\title{
Are Serum Vitamin D Levels Associated With Dry Eye Disease? Results From the Study Group for Environmental
}

\section{Eye Disease}

\author{
Da-Hye Jeon ${ }^{1}$, Hyungseon Yeom ', Jaewon Yang ${ }^{2}$, Jong Suk Song ${ }^{3}$, Hyung Keun Lee ${ }^{4}$, Hyeon Chang Kim ${ }^{1,5}$ \\ ${ }^{1}$ Department of Preventive Medicine, Yonsei University College of Medicine, Seoul; ${ }^{2}$ Department of Public Health, Yonsei University Graduate School, \\ Seoul; ${ }^{3}$ Department of Ophthalmology, Korea University College of Medicine, Seoul; Institute of Vision Research, Department of Ophthalmology, \\ Yonsei University College of Medicine, Seoul; ${ }^{5}$ Cardiovascular and Metabolic Diseases Etiology Research Center, Yonsei University College of Medicine, \\ Seoul, Korea
}

Objectives: Dry eye disease (DED) is an increasingly important public health problem in Korea. Previous studies conducted in Korea have reported inconsistent results regarding the protective effects of vitamin D on DED, and these discrepancies may be related to the relatively simple questionnaire that has been used. Thus, we evaluated the association of serum vitamin D levels with DED using the ocular surface disease index (OSDI).

Methods: The present study evaluated data from participants in the Study Group for Environmental Eye Disease (2014-2015). This group included data from 752 participants, and data from 740 participants (253 men and 487 women) were analyzed in the present study. DED severity was evaluated using the OSDI.

Results: Higher serum vitamin D levels were associated with a non-significantly reduced risk of DED in the crude analysis (odds ratio [OR], $0.991 ; 95 \%$ confidence interval [Cl], 0.971 to 1.011$)$ and in the adjusted analysis (OR, $0.988 ; 95 \% \mathrm{Cl}, 0.966$ to 1.010$)$. In the crude analysis of no/mild DED vs. moderate/severe DED, men exhibited a decreased risk with increasing serum vitamin D levels (OR, 0.999; $95 \% \mathrm{Cl}, 0.950$ to 1.051), while women exhibited an increased risk (OR, 1.003; $95 \% \mathrm{Cl}, 0.979$ to 1.027$)$. In these analyses, we found no significant associations.

Conclusions: The findings of the present study support previous reports that serum vitamin D levels are not associated with DED.

Key words: Dry eye syndromes, Keratoconjunctivitis sicca, Vitamin D, 25-Hydroxyvitamin D2, Korea

\section{INTRODUCTION}

Received: June 7, 2017 Accepted: October 20, 2017

Corresponding author: Hyeon Chang Kim, MD, PhD

50-1 Yonsei-ro, Seodaemun-gu, Seoul 03722, Korea

Tel: +82-2-2228-1873, Fax: +82-2-392-8133

E-mail: hckim@yuhs.ac

This is an Open Access article distributed under the terms of the Creative Commons Attribution Non-Commercial License (http://creativecommons.org/licenses/by$\mathrm{nc} / 4.0 / /$ which permits unrestricted non-commercial use, distribution, and reproduction in any medium, provided the original work is properly cited.
Dry eye disease (DED) is a tear film disorder caused by chronic inflammation of the lacrimal functional unit [1]. DED is one of the most common complaints of patients who visit an ophthalmologist, and most patients with this condition experience symptoms including ocular discomfort, pruritus, redness, pain, and eye fatigue [1-3]. DED is also becoming an increasingly important public health problem because it affects vision-related quality of life (e.g., through adverse effects on 
reading and driving ability) [2-4]. Moreover, the prevalence of DED is increasing worldwide, and its prevalence is higher among Asian populations than among Western populations [5-10]. A Korean study reported that the prevalence of DED was $8.0 \%$ based on physician diagnoses and $14.4 \%$ based on DED symptoms [11].

Recent studies have suggested that vitamin $D$ can protect against DED [12-15]. Vitamin D is a fat-soluble vitamin that is produced by cutaneous synthesis after sun exposure [16], and it has well-known musculoskeletal functions in the cartilage and bone $[17,18]$. Vitamin $D$ has also been reported to have protective effects against various health problems, such as cardiovascular disease (CVD), diabetes, malignancies [19-22], and some eye disorders $[23,24]$. Some Korean studies have evaluated the associations of serum vitamin D levels with DED using Korea National Health and Nutrition Examination Survey (KNHANES) data, but their results were inconsistent [25-27]. They used a simple questionnaire about dry eye syndromes [28]. Therefore, we investigated the association between serum vitamin $D$ levels and DED among Korean adults based on the ocular surface disease index (OSDI).

\section{METHODS}

The present study evaluated data from the Study Group for Environmental Eye Disease (SEED). The SEED study is an ancillary study of the Cardiovascular and Metabolic Disease Etiology Research Center (CMERC) cohort. The CMERC study is an ongoing cohort study (started in 2013) investigating risk factors for CVD and metabolic disease among the general population of healthy community-dwelling Korean adults ( $\geq 30$ years old) [29]. The SEED study is an ongoing multidisciplinary study (started in 2013) that evaluates diagnostic and therapeutic biomarkers for environmental eye diseases. The present study analyzed data that were collected during 2014-2015 from the SEED study (752 participants), although we excluded 12 participants because they had missing data for relevant variables. Thus, we analyzed data from 740 participants ( 253 men and 487 women). The protocol of the present study was approved by the institutional review board of Severance Hospital (Yonsei University College of Medicine). All participants provided informed consent before participating in the CMERC and SEED studies.

In the present study, DED was defined as an OSDI score of $\geq 13.0$. The OSDI uses a DED-specific questionnaire that was developed by the Outcomes Research Group at Allergan, and consists of questions regarding vision-related function (4 questions), eye symptoms (5 questions), and environmental triggers related to DED (3 questions). Each question is scored from 0 to 4 , and the total OSDI score is calculated using the following formula: OSDI= ([sum of scores for all questions answered] $\times 100) /([$ total number of questions answered] $\times 4$ ), with results on a scale of 0 to 100 . No DED (i.e., normal status) is defined as an OSDI score of $<13.0$. The severity of DED is defined as mild (scores of 13.0-22.9), moderate (23.0-32.9), and severe (scores of 33.0-100.0). Previous studies have confirmed that the OSDI is a valid and reliable tool for measuring DED symptoms and their effects on vision-related quality of life $[30,31]$. Trained interviewers individually administered the questionnaires to collect data regarding the participants' demographic information, medical history, and OSDI.

Serum vitamin D levels (25-hydroxyvitamin D) were assessed using a chemiluminescence immunoassay (Liason, DiaSorin, Saluggia, Italy). Peripheral blood samples were obtained after an overnight fast, and all bioassays were performed at a central laboratory (Seoul Clinical Laboratories R\&D Center, Seoul, Korea). Concentrations of blood glucose were measured using a colorimetric analyzer, while lipid profiles (including total cholesterol and high-density lipoprotein [HDL] cholesterol) were analyzed enzymatically. Glycated hemoglobin ( $\mathrm{HbA} 1 \mathrm{c}$ ) concentrations were measured using high-performance liquid chromatography. Height was measured using a stadiometer (DS-102; Jenix, Seoul, Korea), and body weight was measured using a digital scale (DB-150; CAS, Seongnam, Korea). Body mass index (BMI) was calculated as body weight divided by height squared $\left(\mathrm{kg} / \mathrm{m}^{2}\right)$. Systolic and diastolic blood pressures were measured 3 times in the right arm, and the average values of the second and third measurements were used for the analysis [29].

Previous studies have revealed that the major risk factors or confounders for DED include age, women, BMI, smoking, history of eye surgery, diabetes mellitus, visual display terminal (VDT) use, contact lens use, and education level $[10,32,33]$. Thus, when available, we included these variables in our multivariate analyses. Diabetes mellitus was defined as a fasting blood glucose concentration of $\geq 126 \mathrm{mg} / \mathrm{dL}$, an HbA1c concentration of $\geq 6.5 \%$, or the current use of oral medication or insulin injection. Participants were classified according to smoking status as never smokers, former smokers, or current smokers. Because the effect sizes varied according to gender, 
we performed analyses of all participants together and of men and women separately. Continuous and normally distributed variables were compared using the $t$-test, and non-normally distributed continuous variables were compared using the Wilcoxon rank-sum test. Categorical variables were compared using the chi-square test. All tests were 2-tailed, and differences were considered statistically significant at $p$-values of $<0.05$. The associations of serum vitamin $D$ levels with DED were evaluated using logistic regression analyses. Model 1 was not adjusted for any variables, model 2 was adjusted for age and BMI, and model 3 was adjusted for age, BMI, systolic/diastolic blood pressure, total cholesterol, HDL cholesterol, fasting glucose concentration, contact lens use, VDT use, history of eye surgery, and education level. The results of the logistic regression analyses were reported as odds ratios (ORs) with
95\% confidence intervals (Cls). All analyses were performed using SAS version 9.4 (SAS Institute Inc., Cary, NC, USA).

\section{RESULTS}

Table 1 shows the participants' characteristics. As defined by an OSDI score of $\geq 13.0$, the prevalence of DED among the 740 participants was $53.1 \%$. The prevalence of DED among women was significantly higher than among men (58.7 vs. $42.3 \%$, respectively; $p<0.01$ ). The median age was 53.0 years (51.0 years for men and 54.0 years for women). The univariate analyses revealed significant gender-related differences in the values for serum vitamin D, age, BMI, systolic blood pressure, diastolic blood pressure, HDL cholesterol, triglycerides, fasting glucose, history of hypertension, VDT use for $>4$ hours, smok-

Table 1. General characteristics of the participants according to gender

\begin{tabular}{|c|c|c|c|c|}
\hline Variables & Total $(n=740)$ & Men $(n=253)$ & Women $(n=487)$ & $p$-value \\
\hline OSDI score & $14.6[6.3,27.1]$ & $10.7[4.2,20.8]$ & $16.7[8.3,29.5]$ & $<0.01$ \\
\hline Age (y) & $53.0[42.0,58.0]$ & $51.0[39.0,58.0]$ & $54.0[45.0,58.0]$ & 0.05 \\
\hline Triglycerides (mg/dL) & $107.5[79.0,155.0]$ & $132.0[95.0,186.0]$ & $98.0[73.0,134.0]$ & $<0.01$ \\
\hline Fasting glucose (mg/dL) & $88.0[82.0,95.0]$ & $90.0[84.0,99.0]$ & $87.0[81.0,93.0]$ & $<0.01$ \\
\hline $\mathrm{HbA1c}(\%)$ & $5.6[5.4,5.9]$ & $5.6[5.4,5.9]$ & $5.6[5.3,5.9]$ & 0.40 \\
\hline Serum vitamin $\mathrm{D}$ (ng/mL) & $13.5[10.4,18.6]$ & $14.3[10.9,18.5]$ & $13.1[10.0,18.6]$ & 0.03 \\
\hline Body mass index $\left(\mathrm{kg} / \mathrm{m}^{2}\right)$ & $23.6 \pm 3.1$ & $24.6 \pm 3.0$ & $23.1 \pm 3.0$ & $<0.01$ \\
\hline Systolic blood pressure (mmHg) & $117.9 \pm 14.6$ & $125.0 \pm 12.8$ & $114.3 \pm 14.2$ & $<0.01$ \\
\hline Diastolic blood pressure $(\mathrm{mmHg})$ & $74.8 \pm 9.5$ & $79.8 \pm 9.3$ & $72.2 \pm 8.5$ & $<0.01$ \\
\hline Total cholesterol (mg/dL) & $197.9 \pm 34.7$ & $195.8 \pm 34.9$ & $199.0 \pm 34.6$ & 0.24 \\
\hline HDL cholesterol (mg/dL) & $57.9 \pm 14.7$ & $51.1 \pm 12.4$ & $61.4 \pm 14.6$ & $<0.01$ \\
\hline Diabetes & $72(9.7)$ & $29(11.5)$ & $43(8.8)$ & 0.31 \\
\hline Hypertension & $163(22.0)$ & $78(30.8)$ & $85(17.5)$ & $<0.01$ \\
\hline Contact lens use & $20(2.7)$ & $6(2.4)$ & $14(2.9)$ & 0.81 \\
\hline History of eye surgery & $103(13.9)$ & $35(13.6)$ & $68(13.8)$ & \\
\hline VDT use (>4 hr) & $198(26.8)$ & $112(44.3)$ & $86(17.7)$ & $<0.01$ \\
\hline Smoking status (\%) & & & & $<0.01$ \\
\hline Never smoker & $512(69.2)$ & $63(24.9)$ & 449 (92.2) & \\
\hline Former smoker & $127(17.2)$ & $103(40.7)$ & $24(4.9)$ & \\
\hline Current smoker & $101(13.6)$ & $87(34.4)$ & $14(2.9)$ & \\
\hline Education level & & & & $<0.01$ \\
\hline$\leq$ High-school graduate & $379(51.2)$ & $92(36.4)$ & $287(58.9)$ & \\
\hline > High-school graduate & $361(48.8)$ & $161(63.6)$ & $200(41.1)$ & \\
\hline Severity of DED (OSDI score) & & & & $<0.01$ \\
\hline No DED $(<13.0)$ & $347(46.9)$ & $146(57.7)$ & $201(41.3)$ & \\
\hline Mild DED (13.0-22.9) & $169(22.8)$ & $57(22.5)$ & $112(23.0)$ & \\
\hline Moderate DED (23.0-32.9) & $93(12.6)$ & $22(8.7)$ & $71(14.6)$ & \\
\hline Severe DED (33.0-100.0) & $131(17.7)$ & $28(11.1)$ & $103(21.1)$ & \\
\hline
\end{tabular}

Values are presented as mean \pm standard deviation, median [interquartile range], or number (\%).

OSDI, ocular surface disease index; HbA1C, glycosylated hemoglobin; HDL, high-density lipoprotein; VDT, visual display terminal; DED, dry eye disease. 

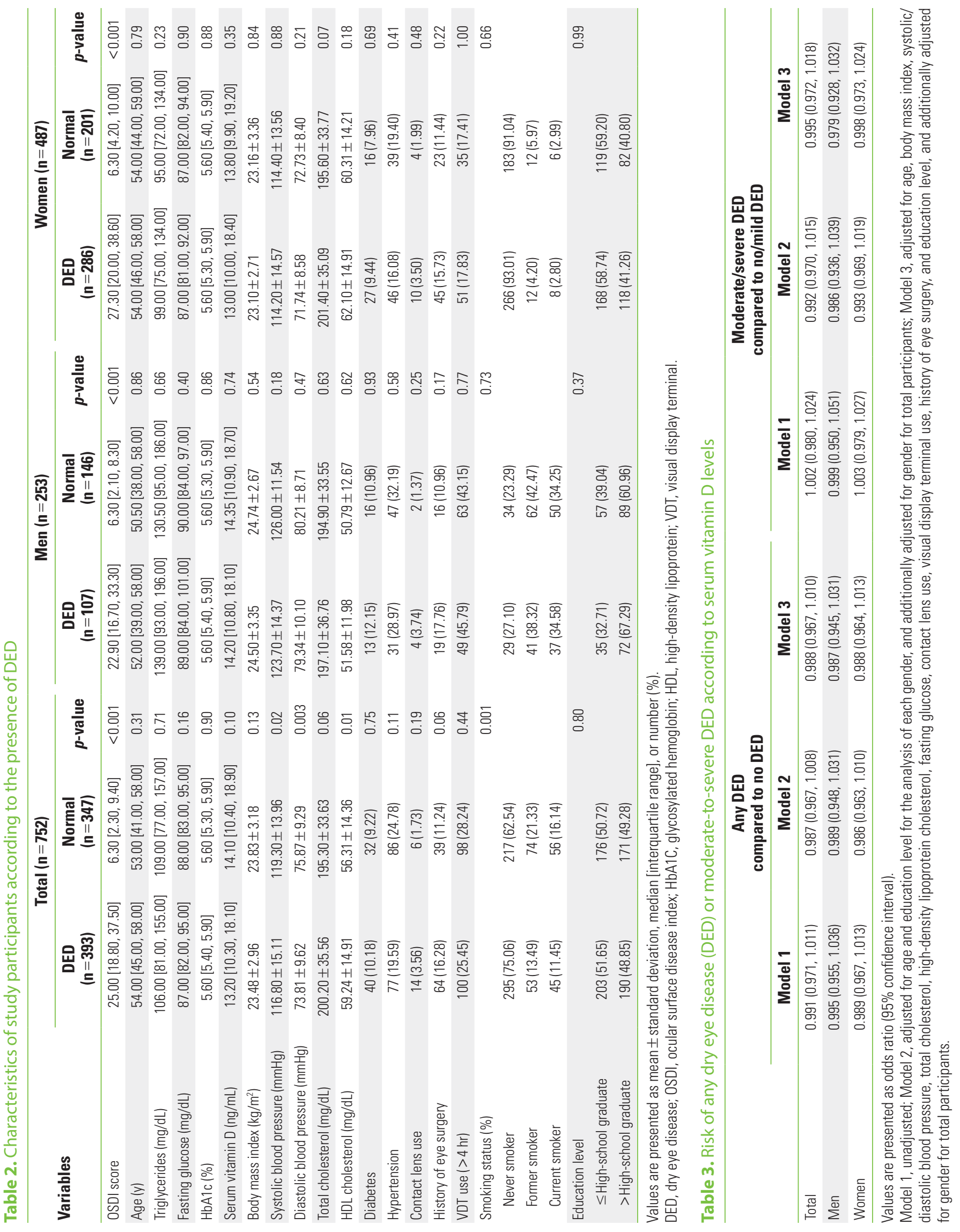
ing status, and education level. No gender-related differences were observed in the values for total cholesterol, $\mathrm{HbA1c}$, history of diabetes mellitus, or contact lens use.

The participants' characteristics according to DED status are shown in Table 2. Serum vitamin D levels were slightly higher among individuals without DED than among individuals with DED, and this non-significant association was observed among all participants $(p=0.10)$, among men $(p=0.74)$, and among women ( $p=0.35$ ). The only significant differences in the overall analyses were observed in the values for systolic blood pressure, diastolic blood pressure, and HDL cholesterol among all participants.

Higher serum vitamin D levels were associated with a nonsignificant reduction in the risk of DED in the crude analysis (OR, $0.991 ; 95 \% \mathrm{Cl}, 0.971$ to 1.011 ) and in the fully adjusted analysis (OR, $0.988 ; 95 \% \mathrm{Cl}, 0.967$ to 1.010$)$ among all participants (Table 3). Among men, the crude OR was $0.995(95 \% \mathrm{Cl}$, 0.955 to 1.036$)$ and the adjusted OR was $0.987(95 \% \mathrm{Cl}, 0.945$ to 1.031$)$. Among women, the crude OR was $0.989(95 \% \mathrm{Cl}$, 0.967 to 1.013$)$ and the adjusted OR was $0.988(95 \% \mathrm{Cl}, 0.964$ to 1.013 ).

When we compared individuals with no/mild DED to individuals with moderate/severe DED, we observed that higher serum vitamin $D$ levels were associated with a non-significant increase in the risk of moderate/severe DED in the crude analysis (OR, 1.002; $95 \% \mathrm{Cl}, 0.980$ to 1.024$)$ and a non-significant decrease in the fully adjusted analysis (OR, 0.995; $95 \% \mathrm{Cl}, 0.972$ to 1.018). We found a non-significant decrease in the risk of moderate/severe DED among men (OR, 0.999; 95\% Cl, 0.950 to 1.051$)$ and a non-significant increase in the risk among women (OR, 1.003; 95\% Cl, 0.979 to 1.027) in the crude analysis. These associations remained non-significant after adjusting for all covariates. In the adjusted analysis, the OR among women was 0.998 ( $95 \% \mathrm{Cl}, 0.973$ to 1.024) (Table 3).

\section{DISCUSSION}

The prevalence of DED in the present study was $53.1 \%$ (42.3\% among men and $58.7 \%$ among women). When we considered only moderate/severe DED, the prevalence was $30.3 \%$ (19.8\% among men and 35.7\% among women). Previous studies have revealed that the prevalence of DED has risen from 4.3 to $73.5 \%$ [5], and our results are within this range. We suggest that the diagnosis of mild DED could cause the prevalence of DED to be overestimated.
In addition, we observed various gender-related differences in the participants' characteristics, and similar results have been observed in a previous study [26]. Some researchers have reported findings that may explain the gender-related differences, which could be related to differences in sex hormones or sunlight exposure times between men and women $[26,34]$. In the present study, our findings may suggest that women are more sensitive to the symptoms of DED. When comparing individuals with no DED to those with DED of any severity, the protective effect of vitamin $D$ was $<1.00$, whereas this effect was $>1.00$ when individuals with no/mild DED were compared to those with moderate/severe DED. This may have been because women were more sensitive to the symptoms. When we compared participants with no/mild DED to those with moderate/severe DED in the crude analyses, we observed opposite directions of the associations of DED with elevated serum vitamin D levels among men and women.

The results of some studies have suggested that vitamin D levels can affect the immune system, and studies in mice have shown that vitamin D enhanced corneal epithelial barrier function through tight or gap junctions $[12,13,35]$. Moreover, vitamin $\mathrm{D}$ can control ocular surface inflammation by inhibiting Langerhans cell migration and corneal neovascularization $[36,37]$. In humans, researchers have reported that vitamin D supplementation is an effective and useful treatment for patients with DED [38]. Our results suggest that oral vitamin D supplementation is less likely to be effective than topical supplementation.

In our study, no association was found between serum vitamin D levels and DED. One possible explanation for this is that it may be difficult for serum vitamin $D$ to reach the cornea because of its lack of vasculature. Another possibility is a positive relationship between aging and serum vitamin D levels. A previous study reported that the aged in Korea and Thailand had higher serum vitamin D levels than the young. They suggested that the elderly had more free time for outdoor activities and tended to have outdoor jobs, such as being farmers or fishermen. Moreover, younger people may use more sunblock than the elderly $[26,39,40]$.

Previous studies conducted in Korea have evaluated the association of serum vitamin D with DED using KNHANES data, which were obtained using the participants' responses to a questionnaire ("Have you ever been diagnosed with DED by an ophthalmologist?" and "Have you experienced symptoms of DED, such as dryness or irritation?") [28]. However, these 
studies have reported inconsistent findings, as some researchers have defined DED based on an ophthalmological diagnosis, while others considered both an ophthalmological diagnosis and DED symptoms. For example, Jee et al. [26] reported that the risk of DED was lower in higher serum vitamin $D$ quintiles (quintiles 5 vs. 1 : $\mathrm{OR}, 0.85 ; 95 \% \mathrm{Cl}, 0.55$ to $1.30 ; p$ for trend $=0.08$ ), although this association was not statistically significant. Yoon et al. [25] also reported that low serum vitamin D levels were a risk factor for DED $(\mathrm{OR}, 1.158 ; 95 \% \mathrm{Cl}, 1.026$ to 1.308), and Kim et al. [27] reported an elevated crude risk of DED at low serum vitamin D levels $(\mathrm{OR}, 1.61 ; 95 \% \mathrm{Cl}, 1.00$ to $2.61 ; p=0.04)$, although this association was attenuated after adjusting for covariates (OR, 1.42; $95 \% \mathrm{Cl}, 0.84$ to 2.40 ). However, the rate of DED diagnosis is relatively low, and its prevalence could be underestimated if it is based on the ophthalmological diagnosis rate, or overestimated if it is based on DED symptoms, which are shared with other eye diseases.

The present study used OSDI scores to identify cases of DED, as this tool is valid and reliable for measuring DED symptoms and their effects on vision-related quality of life [30,31]. We also compared individuals with and without DED, or with no/ mild DED versus moderate/severe DED, because mild symptoms may lead to a misclassification of DED. These analyses failed to detect a significant association between serum vitamin D levels and DED, and our findings support previous reports of no significant association between these variables. This absence of a significant association may be related to the possibility that serum vitamin D levels do not reflect the levels in lacrimal fluid. It is also possible that species-based or racial differences affect these associations, as previous studies regarding the protective effect of vitamin D were conducted in mouse models $[12,13,35]$.

The present study has some limitations that warrant consideration. First, the cross-sectional design precludes any conclusions regarding causality, although previous studies conducted in Korea have also used cross-sectional designs. Second, our dataset was relatively limited compared to the KNHANES data set, which may limit the representativeness of our findings. Nevertheless, our use of the OSDI to detect DED likely provided more detailed and objective information regarding DED severity. Third, we did not consider seasonal variation in serum vitamin D levels. To deal with this variation, previous studies using KNHANES data adjusted for sunlight exposure or occupation. In the KNHANES, information about sunlight exposure and occupation was acquired through the question- naire, and the surveys were conducted throughout the year. Our data were gathered from October 2014 to January 2015 and from October 2015 to December 2015. Thus, our data are likely to contain less seasonal variation than KNHANES.

The present study provides epidemiological data regarding the absence of an association of serum vitamin D levels and DED in the Korean general population. This result is consistent with the findings of Jee et al. [26], who analyzed KNHANES data. Further studies are needed to investigate the mechanisms and management of DED.

\section{ACKNOWLEDGEMENTS}

This study was funded by the Korean Health Technology $\mathrm{R} \& \mathrm{D}$ Project (HI13C0055).

\section{CONFLICT OF INTEREST}

The authors have no conflicts of interest associated with the material presented in this paper.

\section{ORCID}

Da-Hye Jeon https://orcid.org/0000-0002-7913-8357

Hyungseon Yeom https://orcid.org/0000-0002-8004-8244

Hyeon Chang Kim https://orcid.org/0000-0001-7867-1240

\section{REFERENCES}

1. Stern ME, Beuerman RW, Fox RI, Gao J, Mircheff AK, Pflugfelder SC. The pathology of dry eye: the interaction between the ocular surface and lacrimal glands. Cornea 1998;17(6): 584-589.

2. Miljanović B, Dana R, Sullivan DA, Schaumberg DA. Impact of dry eye syndrome on vision-related quality of life. Am J Ophthalmol 2007;143(3):409-415.

3. Tong L, Waduthantri S, Wong TY, Saw SM, Wang JJ, Rosman M, et al. Impact of symptomatic dry eye on vision-related daily activities: the Singapore Malay Eye Study. Eye (Lond) 2010; 24(9):1486-1491.

4. Le Q, Zhou X, Ge L, Wu L, Hong J, Xu J. Impact of dry eye syndrome on vision-related quality of life in a non-clinic-based general population. BMC Ophthalmol 2012;12:22.

5. Um SB, Kim NH, Lee HK, Song JS, Kim HC. Spatial epidemiology of dry eye disease: findings from South Korea. Int J Health 
Journal of

Geogr 2014;13:31.

6. Schaumberg DA, Sullivan DA, Buring JE, Dana MR. Prevalence of dry eye syndrome among US women. Am J Ophthalmol 2003;136(2):318-326.

7. Schaumberg DA, Dana R, Buring JE, Sullivan DA. Prevalence of dry eye disease among US men: estimates from the Physicians' Health Studies. Arch Ophthalmol 2009;127(6):763-768.

8. Viso E, Rodriguez-Ares MT, Gude F. Prevalence of and associated factors for dry eye in a Spanish adult population (the Salnes Eye Study). Ophthalmic Epidemiol 2009;16(1):15-21.

9. Jie Y, Xu L, Wu YY, Jonas JB. Prevalence of dry eye among adult Chinese in the Beijing Eye Study. Eye (Lond) 2009;23(3):688693.

10. Uchino M, Nishiwaki Y, Michikawa T, Shirakawa K, Kuwahara E, Yamada $M$, et al. Prevalence and risk factors of dry eye disease in Japan: Koumi study. Ophthalmology 2011;118(12):23612367.

11. Ahn JM, Lee SH, Rim TH, Park RJ, Yang HS, Kim TI, et al. Prevalence of and risk factors associated with dry eye: the Korea National Health and Nutrition Examination Survey 2010-2011. Am J Ophthalmol 2014;158(6):1205-1214.

12. Yin Z, Pintea V, Lin Y, Hammock BD, Watsky MA. Vitamin D enhances corneal epithelial barrier function. Invest Ophthalmol Vis Sci 2011;52(10):7359-7364.

13. Elizondo RA, Yin Z, Lu X, Watsky MA. Effect of vitamin D receptor knockout on cornea epithelium wound healing and tight junctions. Invest Ophthalmol Vis Sci 2014;55(8):5245-5251.

14. Reins RY, Hanlon SD, Magadi S, McDermott AM. Effects of topically applied vitamin D during corneal wound healing. PLoS One 2016;11(4):e0152889.

15. Lin Y, Ubels JL, Schotanus MP, Yin Z, Pintea V, Hammock BD, et al. Enhancement of vitamin $D$ metabolites in the eye following vitamin D3 supplementation and UV-B irradiation. Curr Eye Res 2012;37(10):871-878.

16. Matsuoka LY, Wortsman J, Hollis BW. Suntanning and cutaneous synthesis of vitamin D3. J Lab Clin Med 1990;116(1):8790.

17. Knutsen KV, Brekke M, Gjelstad S, Lagerløv P. Vitamin D status in patients with musculoskeletal pain, fatigue and headache: a cross-sectional descriptive study in a multi-ethnic general practice in Norway. Scand J Prim Health Care 2010;28(3):166171.

18. Knutsen KV, Madar AA, Brekke M, Meyer HE, Natvig B, Mdala I, et al. Effect of vitamin $D$ on musculoskeletal pain and headache: a randomized, double-blind, placebo-controlled trial among adult ethnic minorities in Norway. Pain 2014;155(12): 2591-2598.

19. Norman PE, Powell JT. Vitamin D and cardiovascular disease. Circ Res 2014;114(2):379-393.

20. Chiu KC, Chu A, Go VL, Saad MF. Hypovitaminosis D is associated with insulin resistance and beta cell dysfunction. Am J Clin Nutr 2004;79(5):820-825.

21. Pereira F, Larriba MJ, Muñoz A. Vitamin D and colon cancer. Endocr Relat Cancer 2012;19(3):R51-R71.

22. Osborne JE, Hutchinson PE. Vitamin D and systemic cancer: is this relevant to malignant melanoma? Br J Dermatol 2002;147 (2):197-213.

23. Etemadifar M, Abtahi SH, Razmjoo H, Abtahi MA, Dehghani A, Salari $M$, et al. 25-hydroxyvitamin $D$ concentrations in patients with optic neuritis as a clinically isolated syndrome and healthy controls. Int J Prev Med 2012;3(5):313-317.

24. Yazar S, Hewitt AW, Black LJ, McKnight CM, Mountain JA, Sherwin JC, et al. Myopia is associated with lower vitamin D status in young adults. Invest Ophthalmol Vis Sci 2014;55(7): 4552-4559.

25. Yoon SY, Bae SH, Shin YJ, Park SG, Hwang SH, Hyon JY, et al. Low serum 25-hydroxyvitamin $D$ levels are associated with dry eye syndrome. PLoS One 2016;11(1):e0147847.

26. Jee D, Kang S, Yuan C, Cho E, Arroyo JG; Epidemiologic Survey Committee of the Korean Ophthalmologic Society. Serum 25-hydroxyvitamin D levels and dry eye syndrome: differential effects of vitamin D on ocular diseases. PLoS One 2016;11 (2):e0149294.

27. Kim MJ, Hwang HR, Kim YJ, Lee SY, Lee JG, Jeong DW, et al. Association between serum 25 -hydroxyvitamin $D$ levels and dry eye in Korean adults: a study based on Korean National Health and Nutrition Examination Survey, 2010-2011. Korean J Fam Med 2017;38(2):81-85.

28. Yoon KC, Choi W, Lee HS, Kim SD, Kim SH, Kim CY, et al. An overview of ophthalmologic survey methodology in the 2008-2015 Korean National Health and Nutrition Examination Surveys. Korean J Ophthalmol 2015;29(6):359-367.

29. Shim JS, Song BM, Lee JH, Lee SW, Park JH, Choi DP, et al. Cardiovascular and Metabolic Diseases Etiology Research Center (CMERC) cohort: study protocol and results of the first 3 years of enrollment. Epidemiol Health 2017;39:e2017016.

30. Ozcura F, Aydin S, Helvaci MR. Ocular surface disease index for the diagnosis of dry eye syndrome. Ocul Immunol Inflamm 2007;15(5):389-393.

31. Schiffman RM, Christianson MD, Jacobsen G, Hirsch JD, Reis 
BL. Reliability and validity of the ocular surface disease index. Arch Ophthalmol 2000;118(5):615-621.

32. Moss SE, Klein R, Klein BE. Prevalence of and risk factors for dry eye syndrome. Arch Ophthalmol 2000;118(9):1264-1268.

33. Messmer EM. The pathophysiology, diagnosis, and treatment of dry eye disease. Dtsch Arztebl Int 2015;112(5):71-81.

34. Song JY, Kim MH, Paik JS, Kim HS, Na KS; Epidemiologic Survey Committee of the Korean Ophthalmologic Society. Association between menstrual irregularity and dry eye disease: a population-based study. Cornea 2016;35(2):193-198.

35. Lu X, Watsky MA. Effects of vitamin D receptor knockout on cornea epithelium gap junctions. Invest Ophthalmol Vis Sci 2014;55(5):2975-2982.

36. Suzuki T, Sano Y, Sotozono C, Kinoshita S. Regulatory effects of 1alpha,25-dihydroxyvitamin $\mathrm{D}(3)$ on cytokine production by human corneal epithelial cells. Curr Eye Res 2000;20(2):
127-130.

37. Suzuki T, Sano Y, Kinoshita S. Effects of 1alpha,25-dihydroxyvitamin D3 on Langerhans cell migration and corneal neovascularization in mice. Invest Ophthalmol Vis Sci 2000;41(1):154158.

38. Bae SH, Shin YJ, Kim HK, Hyon JY, Wee WR, Park SG. Vitamin D supplementation for patients with dry eye syndrome refractory to conventional treatment. Sci Rep 2016;6:33083.

39. Choi HS, Oh HJ, Choi H, Choi WH, Kim JG, Kim KM, et al. Vitamin $D$ insufficiency in Korea--a greater threat to younger generation: the Korea National Health and Nutrition Examination Survey (KNHANES) 2008. J Clin Endocrinol Metab 2011;96(3): 643-651.

40. Chailurkit LO, Aekplakorn W, Ongphiphadhanakul B. Regional variation and determinants of vitamin $D$ status in sunshineabundant Thailand. BMC Public Health 2011;11:853. 